\title{
El balance (trade-off) entre los impactos sociales, económicos y ambientales en la evaluación ex-ante de los proyectos de transporte
}

\author{
Paola Carolina Bueno \\ Investigadora, Centro de Investigación del Transporte (TRANSYT), Universidad \\ Politécnica de Madrid, España \\ José Manuel Vassallo \\ Profesor Titular, Departamento de Ingeniería Civil. Transporte y Territorio, \\ Universidad Politécnica de Madrid, España
}

\section{RESUMEN}

A pesar de la existencia de diferentes herramientas, en la actualidad no existe una metodología estandarizada para la evaluación ex-ante de la sostenibilidad en proyectos de infraestructura durante su ciclo de vida. Aunque las técnicas tradicionalmente aceptadas ofrecen un valioso apoyo en la evaluación de los proyectos, abordan muy someramente los tres componentes de sostenibilidad de manera holística. Esta investigación se centra en uno de los métodos más comunes de evaluación y toma de decisiones sobre proyectos de transporte: El análisis de decisión multicriterio (MCDA). A pesar de que este método puede tratar de manera explícita los diferentes componentes de sostenibilidad, aún persisten algunas dificultades fundamentales. Uno de los principales problemas es establecer con precisión los pesos de importancia relativa a los diferentes criterios definidos. Con el objeto de encontrar el balance (trade-off) entre los impactos sociales, económicos y ambientales en la evaluación ex-ante de los proyectos de transporte, esta investigación desarrolla una adaptación al procedimiento clásico de estimación de las prioridades o pesos dentro de un enfoque multicriterio. Para ello, se ha diseñado un modelo de ponderación compuesto que permite la incorporación de juicios y preferencias basadas en el consenso de expertos (utilizando los métodos REMBRANDT y Delphi), junto con la influencia del contexto geográfico y social del proyecto. Esta nueva metodología se aplica con éxito a un caso de estudio real en España. El resultado demuestra que el modelo es significativo y puede ser aplicado como una herramienta útil en un proceso de evaluación multicriterio.

\section{INTRODUCCIÓN}

Desde que surge el concepto de sostenibilidad ha habido un creciente interés en la exploración de estrategias para promover proyectos y políticas de transporte sostenibles. Actualmente el reto consiste en mitigar los impactos negativos en el medio ambiente, en la economía, y en la sociedad a lo largo de todo el ciclo de vida. Aunque existen múltiples enfoques para evaluar la viabilidad socioeconómica y ambiental de los proyectos de 
transporte, la comunidad científica y los tomadores de decisiones aún no cuentan con una metodología estandarizada, comúnmente aceptada y que ofrezca una medición fiable de la sostenibilidad en la evaluación de los proyectos de transporte. La literatura señala que los políticos requieren herramientas y técnicas prácticas para evaluar la sostenibilidad en todas las etapas de vida de los proyectos de infraestructura (Dasgupta, 2005; Gilmour et al., 2011; Tsai y Chang, 2012).

Los enfoques actuales para la evaluación de proyectos se pueden agrupar en tres categorías principales (ver Bueno, Vassallo y Cheung, 2015). La primera comprende las técnicas tradicionales de toma de decisiones, como el análisis de coste-beneficio (ACB), el análisis de decisión multicriterio (MCDA) o el life-cycle assessment (LCA), entre otros. El segundo grupo incluye los sistemas de calificación de la sostenibilidad de las infraestructuras, denominados rating systems; y el tercero abarca los marcos, las directrices y los diferentes modelos utilizados para realizar la evaluación de la sostenibilidad.

Dentro de todas las herramientas existentes, las formas más comunes para la evaluación exante de la sostenibilidad de los proyectos de transporte son el ACB y el MCDA. Aunque estas técnicas son tradicionalmente aceptadas y ofrecen un valioso apoyo para la toma de decisiones, casi nunca abordan todos los componentes de la sostenibilidad (económico, social y ambiental) de manera minuciosa. Esta investigación se centra en uno de los métodos más comunes de evaluación de los proyectos o políticas de transporte: el análisis multicriterio. A pesar de que esta técnica puede tratar explícitamente los diferentes componentes de la sostenibilidad; sigue habiendo algunas lagunas fundamentales que pueden dar lugar a la falta de precisión en los resultados de un proceso de toma de decisiones. Uno de los principales problemas de esta técnica es el ajuste con precisión de los pesos de los criterios de una manera transparente y precisa.

Como una nueva contribución al estado del arte, esta investigación desarrolla una adaptación al proceso de ponderación y asignación de pesos de los diferentes criterios de un tradicional análisis multicriterio. Con este fin, hemos diseñado un modelo de ponderación compuesto que incorpora el juicio y las preferencias de expertos, junto con evaluaciones objetivas del contexto geográfico y social donde se desarrolla el proyecto.

El documento está estructurado de la siguiente manera. La siguiente sección resume la revisión de la literatura sobre el proceso de obtención de pesos en un análisis multicriterio. Por su parte, el nuevo método de adaptación del MCDA se presenta en la sección 2. Finalmente, la sección 3 analiza los resultados de la aplicación de este enfoque en un caso real; y la última sección presenta las conclusiones y discusiones de este trabajo de investigación.

\section{REVISIÓN DE LA LITERATURA}


La técnica multicriterio es una metodología una adecuada para la toma de decisiones, útil para hacer frente a problemas complejos que involucran múltiples criterios, intereses y perspectivas. En el campo de la ingeniería de transporte, varios autores han sugerido que el MCDA es la herramienta más adecuada para adoptar las decisiones sobre la base de una evaluación integrada de la sostenibilidad (Janic, 2003; Tudela et al., 2006). Su uso para diferentes propósitos se ha incrementado a través de los años. Existen varios trabajos donde este enfoque se ha aplicado en el ámbito del transporte, por ejemplo Iniestra y Gutiérrez (2009) y Cheng (2005).

En un análisis completo realizado por Bueno, Vassallo y Cheung (2015) se concluye que entre las herramientas existentes, el MCDA parece ser la técnica más adecuada debido a su flexibilidad para incorporar los tres aspectos de la sostenibilidad de manera simultánea. El MCDA ofrece una serie de ventajas en comparación con otras técnicas convencionales económicas (ver Munda, 1995). Sin embargo, la literatura ha reconocido diferentes inconvenientes de esta técnica como la evaluación inherente subjetiva, la complejidad de la identificación de los impactos y su método de medición, y la obtención de los pesos relacionados con los diferentes criterios involucrados en la evaluación (Browne y Ryan, 2011) .

De hecho, el tema de los pesos o ponderaciones asignados por el agente decisor y que reflejan la importancia o preferencia relativa de cada criterio; es la principal cuestión no resuelta de esta metodología. Ese concepto conocido en la literatura como 'caja negra' provoca una pérdida de credibilidad de la herramienta por tener que ver con la transparencia en los resultados. Debido a: (i) la falta de procedimientos para agregar las evaluaciones de múltiples criterios individuales de diferente naturaleza y (ii) que la obtención de los pesos ha sido tradicionalmente arbitraria y sometida a opiniones subjetivas por parte de los decisores, algunos gobiernos (como Francia) han dispuesto no utilizar el MCDA y regresar al enfoque monetizable (Sayers et al., 2003).

Como resultado, el MCDA ha sido objeto de críticas severas por un gran número de estudios (Browne y Ryan, 2011; Chen et al., 2013; Hobbs y Horn, 1997; Wibowo y Deng, 2011). De acuerdo con la revisión literaria, a pesar de algunos avances y nuevas técnicas propuestas ver por ejemplo Wang (2015) y Rezaei (2015) - es evidente que sigue habiendo un margen de mejora muy significativo para el proceso de asignación de los pesos a los criterios. Algunos de los nuevos enfoques generalmente requieren herramientas matemáticas complejas o se encuentran sujetos a la subjetividad. Los responsables políticos todavía están en necesidad de métodos normalizados y prácticos para evaluar las ventajas y desventajas entre los aspectos económicos, ambientales y sociales en los proyectos de transporte. Este balance de ponderaciones entre tres componentes constituye una de las más importantes necesidades de investigación que deben emprenderse con el fin de mejorar la evaluación de la sostenibilidad de los proyectos de transporte. La siguiente sección discute un enfoque 
flexible para superar los obstáculos señalados anteriormente.

\section{METODOLOGÍA COMPUESTA PARA LA ASIGNACIÓN DE LA IMPORTANCIA RELATIVA DE CADA CRITERIO DENTRO DE UN ANÁLISIS MULTICRITERIO}

El objetivo de esta sección es presentar una nueva metodología para el establecimiento de los pesos de los diferentes criterios de sostenibilidad, que permita al final una agregación ponderada de acuerdo con la importancia dentro de conjunto de objetivos. La novedad de este método es la consideración, por separado, de las preferencias de expertos y una serie de características objetivas de los criterios que dependen del contexto geográfico y social del proyecto. Los beneficios de este procedimiento propuesto son tres: (i) mayor eficiencia en el establecimiento de las prioridades para los criterios; (ii) mayor rigurosidad en el mecanismo de cálculo de los pesos o índices de ponderación; y (iii) mayor grado de objetividad en el proceso de trade-off.

El modelo compuesto está orientado a la obtención mejores coeficientes de ponderación para los diferentes criterios de sostenibilidad (denominados pesos mejorados: $\mathrm{IW}_{\mathrm{S}}$ ), como se muestra en la Ecuación 1. Los pesos provienen del contexto (nivel de severidad) y de los juicios comparativos basados en el consenso y las preferencias de expertos (pesos convergentes: $\mathrm{CW}_{\mathrm{S}}$ ). Estos términos se explicarán en mayor detalle en las secciones 3.1 y 3.2 .

$$
I W_{i}=C W_{i} \times S L_{i}
$$

Donde,

$I W_{i}$ : Peso mejorado para el criterio $i$;

$\mathrm{CW}_{i}$ : Peso convergente para el criterio $i$;

$S L_{i}: \quad$ Nivel de severidad del criterio $i$.

\subsection{Peso convergente para los criterios}

Con el objetivo reducir la brecha entre la teoría y la práctica y de combinar lo científico y racional con lo intangible, se considera crucial que en un análisis multicriterio los pesos asignados incorporen las preferencias de los tomadores de decisiones (Gühnemann et al., 2012). Bajo esta premisa, proponemos como primer paso el uso conjunto de la metodología REMBRANDT (por sus siglas en inglés Ratio Estimation in Magnitudes or deciBells to Rate Alternatives which are Non-DominaTed system) y el método Delphi.

Como primer paso para determinar los pesos de cada criterio con el fin de establecer la importancia relativa entre los diferentes impactos a considerar en una evaluación de sostenibilidad, se plantea un método de comparación dos a dos (denominado en inglés pairwise comparison method). Para ello, utilizamos la técnica de Rembrandt para calcular 
las ponderaciones. Además, dado que el consenso es rara vez alcanzado en este tipo de procesos analíticos jerárquicos, proponemos también emplear la técnica Delphi (ver Linstone y Turoff, 1975) con el propósito de lograr una convergencia en la opinión de los expertos. El proceso completo se recoge en la Figura 1.

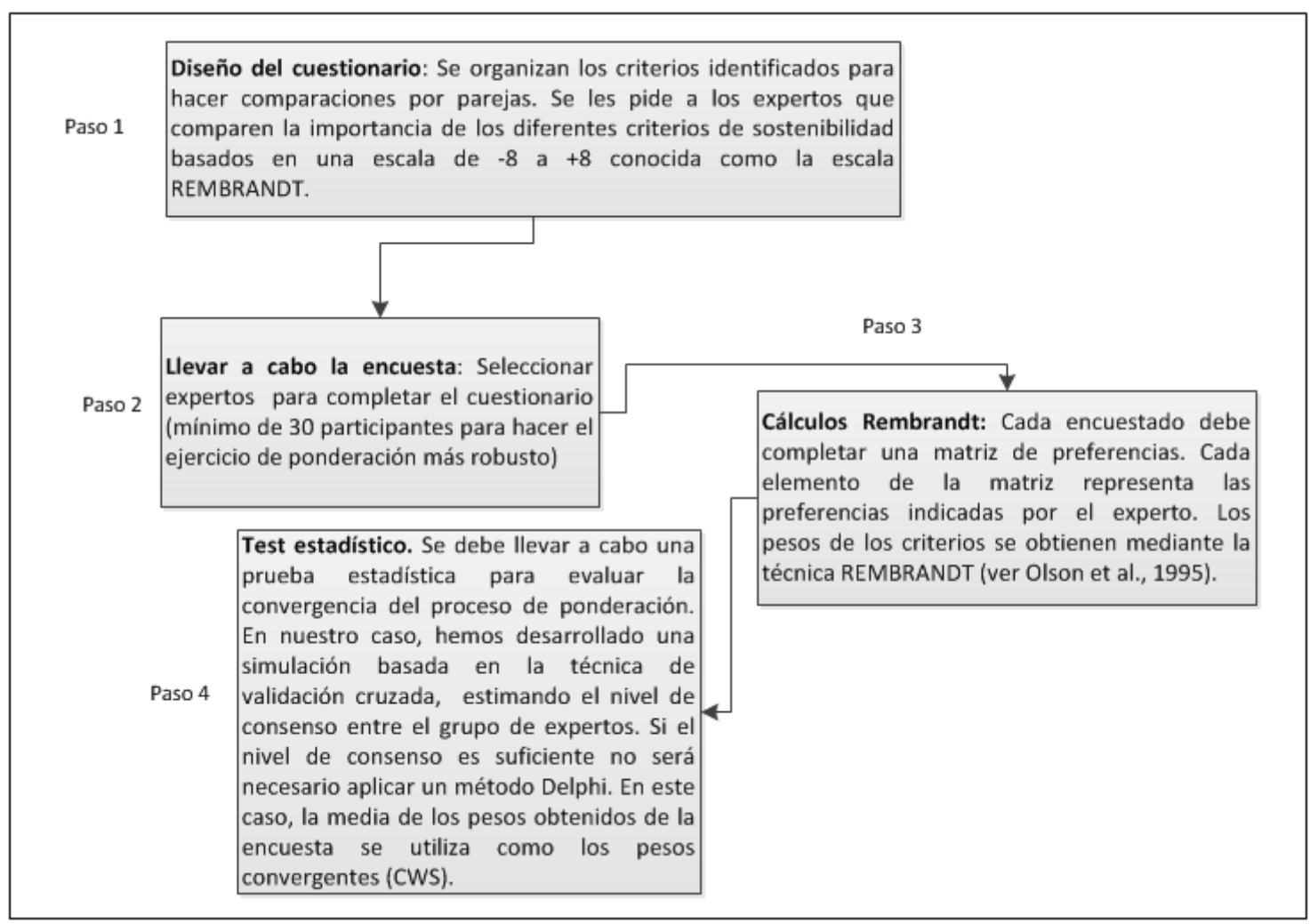

Fig. 1 - Pasos para obtener los pesos convergentes de los criterios

\subsection{Nivel de severidad para los criterios}

El propósito de este paso es establecer objetivamente los aspectos relacionados con la sensibilidad geográfica y el contexto social en el que se enmarca el proyecto. En nuestra metodología, los niveles de severidad (SL) para cada criterio se deben obtener mediante una agregación de puntajes obtenidos tras la evaluación de la situación actual o presente (PS) y la tendencia en el contexto del proyecto. Cuanto mayor sea el SL, más sensible será el criterio en el contexto. Como se muestra en la Ecuación 1, los SL se integran en el proceso de evaluación al considerarlos parte del método de ponderación.

La integración del contexto debe ser independiente de las magnitudes de los impactos e incluso del proyecto por sí mismo (para evitar la doble contabilización). La primera subtarea consiste en la identificación de la importancia relativa de cada criterio para la evaluación de la sostenibilidad en el contexto determinado. La PS de cada criterio debe ser evaluada utilizando una escala definida. El decisor debe comparar el valor para el contexto con un valor razonable conocido para este criterio específico (que se define como el valor 
medio para otros contextos similares o promedio en el contexto). Lo anterior, permite a los responsables políticos evaluar comparativamente el desempeño ambiental, económico y social de la región donde el proyecto se llevará a cabo con otros países con características geográficas, sociales o regionales similares.

Es probable que el criterio tenga un mayor impacto en la sostenibilidad global si el PS se considera "mucho peor" que el valor razonable conocido. Por lo tanto, en nuestra metodología, una puntuación es asignada a la PS para cada criterio en el contexto de acuerdo con la Tabla 1. En estos casos, lo que es mucho/poco/ moderadamente mejor o peor debería guiarse por la legislación o por lo que indique la disciplina particular de los criterios evaluados.

\begin{tabular}{|l|l|}
\hline $\begin{array}{c}\text { Puntos asignados a } \\
\text { la PS de cada } \\
\text { atributo }\end{array}$ & \multicolumn{2}{|c|}{ Descripción } \\
\hline 0 puntos & $\begin{array}{l}\text { Si la PS del atributo es considerada mucho mejor o } \\
\text { moderadamente mejor que el promedio en el contexto. }\end{array}$ \\
\hline 1 punto & $\begin{array}{l}\text { Si la PS del atributo es considerada ligeramente mejor el promedio } \\
\text { en el contexto. }\end{array}$ \\
\hline 2 puntos & $\begin{array}{l}\text { Si la PS del atributo es considerada similar el promedio en el } \\
\text { contexto. }\end{array}$ \\
\hline 3 puntos & $\begin{array}{l}\text { Si la PS del atributo es considerada ligeramente peor el promedio } \\
\text { en el contexto. }\end{array}$ \\
\hline 4 puntos & $\begin{array}{l}\text { Si la PS del atributo es considerada moderadamente peor el } \\
\text { promedio en el contexto. }\end{array}$ \\
\hline 5 puntos & $\begin{array}{l}\text { Si la PS del atributo es considerada mucho peor el promedio en el } \\
\text { contexto. }\end{array}$ \\
\hline
\end{tabular}

Tabla 1 -Evaluación de la PS para cada atributo

Un breve ejemplo puede servir para ilustrar el proceso de evaluación de la PS presentado en el párrafo anterior. Imaginemos dos proyectos de transporte con las mismas características pero implementados en diferentes países: Alemania y España. Para calcular la situación presente del criterio 'efectos en el empleo' para ambos proyectos, se debe comparar la tasa de desempleo en Alemania y España con el valor medio de este ratio en diferentes países europeos. De acuerdo con la base de datos del Banco Mundial, el porcentaje de la fuerza laboral que está sin trabajo es de 5,3\% en Alemania y de 26\% para España, mientras que el promedio de la tasa de desempleo en los países europeos es de 10,9\%. En el caso del proyecto que se desarrollará en Alemania, podemos suponer razonablemente que la PS para este criterio es mucho mejor que la media de contexto, y 0 puntos deben ser asignados a la PS del criterio de 'efectos en el empleo'. Por el contrario, en el caso de España, la PS es mucho peor y, de acuerdo con la Tabla 1, 5 puntos se deben asignar a este criterio en este contexto. 
Como segunda sub-tarea para calcular el nivel de severidad, nuestra metodología propone evaluar también la tendencia, además de la PS, para cada criterio en el contexto geográfico donde se localizará el proyecto. El principal resultado de esta sub-tarea actual es la clasificación de cada tendencia como "mejora", "estable" o "empeora". La asignación de la puntuación es la siguiente 0 puntos, 1 punto y 2 puntos, respectivamente. Continuando con el ejemplo descrito anteriormente, el porcentaje de la tasa de paro en España en el momento de tramitar el proyecto era creciente, mientras que en Alemania ha ido disminuyendo desde hace muchas décadas. Entonces, para el primer caso, la tendencia del criterio debería ser clasificada como "empeora" y un total de 2 puntos deberían asignarse. En contraste para el caso de Alemania, 0 puntos deben ser asignados. En resumen, el criterio 'efectos en el empleo' es más sensible y tiene un mayor nivel de importancia para un proyecto que se aplique en España y, de acuerdo con la Ecuación 1, deberá tener un peso mayor que el mismo criterio para el proyecto alemán.

El proceso descrito anteriormente para el ítem de 'efectos de empleo' se debe repetir para cada uno de los otros criterios que vayan a ser considerados para la evaluación sostenible del proyecto a lo largo de su ciclo de vida (por ejemplo para los accidentes, los impactos auditivos, los efectos distributivos del proyecto, etc).

\section{UNA BREVE APLICACIÓN DE LA METODOLOGÍA A UN CASO DE ESTUDIO REAL}

Para demostrar la viabilidad y la utilidad de la metodología propuesta, esta sección describe su aplicación a un caso práctico de toma de decisiones en relación con el diseño y la construcción de una nueva carretera interurbana en España.

En primer lugar, se identificaron los criterios apropiados para medir el desempeño de las diferentes alternativas de proyecto teniendo en cuenta el concepto de sostenibilidad, es decir la eficiencia económica, la protección del medio ambiente y los aspectos sociales. La lista de criterios para este caso real, se muestra en la Tabla 2, segunda columna. Además de estos criterios, tuvimos que identificar un atributo en el contexto para evaluar el desempeño de cada criterio. Por ejemplo, para evaluar la importancia del criterio 'costes de inversión', podemos evaluar el atributo 'disponibilidad presupuestaria para el gasto en infraestructura ' en el contexto. La Tabla 2 (tercera columna) también presenta la lista de estos atributos. 


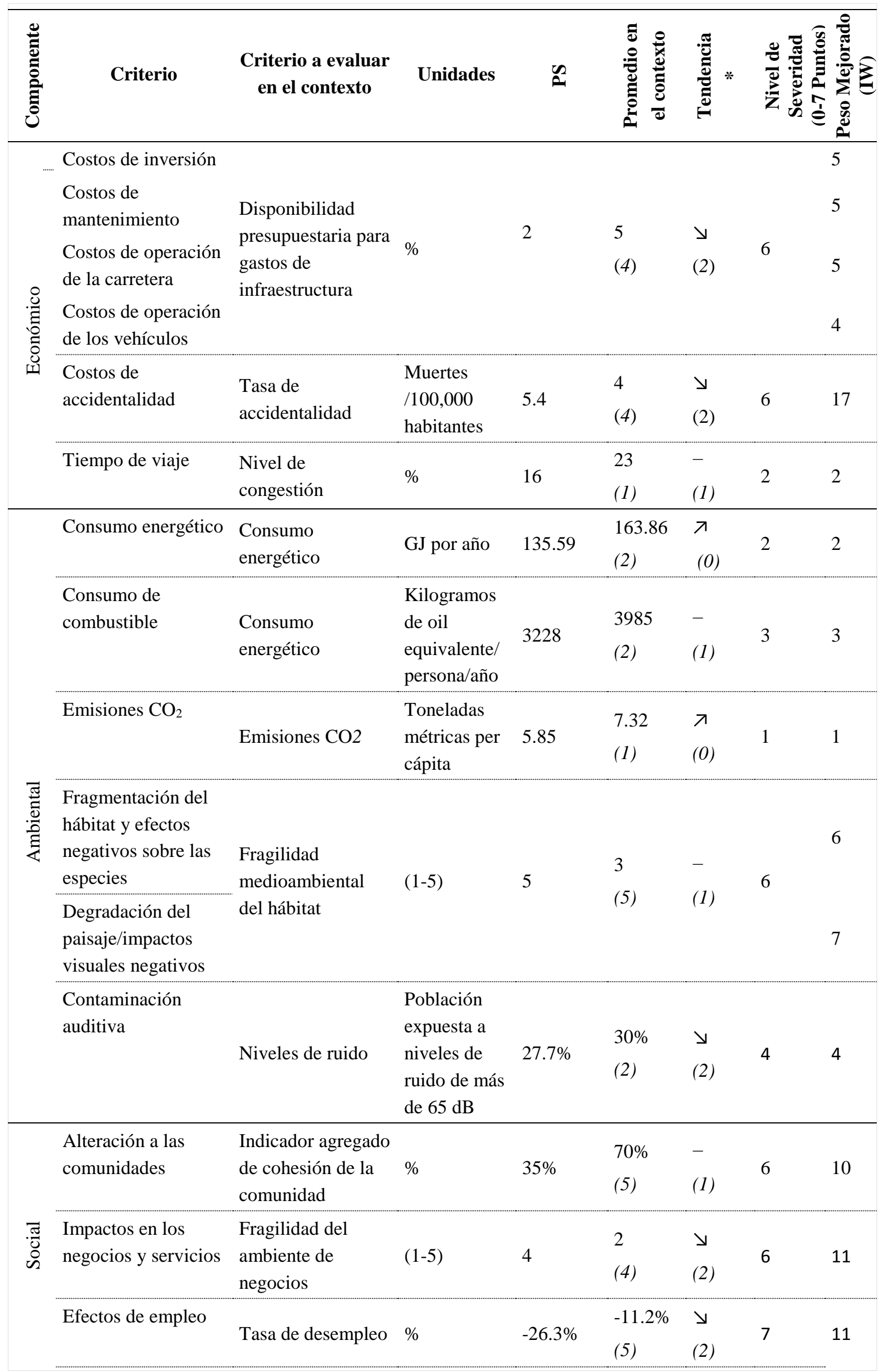




\begin{tabular}{|c|c|c|c|c|c|c|c|}
\hline $\begin{array}{l}\text { Efectos distributivos } \\
\text { del proyecto }\end{array}$ & $\begin{array}{l}\text { Coeficiente de } \\
\text { Gini }\end{array}$ & $\begin{array}{l}\text { Sin } \\
\text { dimensión }\end{array}$ & 0.34 & $\begin{array}{l}0.29 \\
(4)\end{array}$ & $\begin{array}{l}- \\
(1)\end{array}$ & 5 & 8 \\
\hline
\end{tabular}

*De acuerdo con nuestra metodología se clasifica como: mejorando ( $\nearrow$ ), estable (-) o empeorando ( $)$.

\section{Tabla 2 - Situación presente, tendencia, nivel de severidad y peso mejorado para los criterios de sostenibilidad}

Para ayudar a ilustrar mejor cómo incorporar el contexto en el proceso, la tabla presenta una completa la evaluación de la PS y la tendencia para cada criterio en la zona geográfica particular en la que se va a estar ubicado el proyecto (España). Esta información se obtuvo principalmente de la base de datos del Banco Mundial, datos de Eurostat y otras fuentes oficiales. Asignamos una puntuación a la PS para cada criterio en el contexto (que se muestra en cursiva, debajo del 'promedio en el contexto'. A las tendencias para cada criterio particular también se les asignó una puntuación. Finalmente, el nivel de severidad se obtuvo mediante la suma de las calificaciones de la PS y las puntuaciones de la tendencia.

Por ejemplo, en el caso de los `efectos distributivos del proyecto', nos basamos en Eurostat para obtener el coeficiente de Gini (la medida más ampliamente utilizada para medir la desigualdad de ingresos). El valor de este coeficiente en España fue de alrededor de 0,34, mientras que el promedio de los países de la Unión Europea fue de 0,29. Dado que la PS se considera moderadamente peor que el valor promedio en el contexto, una puntuación de 4 puntos fue asignada a este criterio de acuerdo con la Tabla 1. Además, ya que la tendencia para el coeficiente de Gini es estable, se asignó 1 punto a este criterio. Como resultado, se obtuvo un nivel de severidad de 5 puntos para el criterio de 'efectos distributivos del proyecto'. Esto significa que, en el momento de llevar a cabo este análisis, los efectos distributivos del proyecto tienen menos importancia en el contexto de España que, por ejemplo, los efectos en el empleo (ver Tabla 2). Como consecuencia de ello, partiendo del supuesto de que ambos criterios tuvieran un mismo CW y de acuerdo con la Ecuación 1, los efectos sobre el empleo deberían tener un peso final superior.

Como segunda parte del ejercicio, para obtener los pesos de preferencia, organizamos comparaciones por parejas basándonos en la lista de criterios anteriormente identificados. Esto, con el fin de tener en cuenta las opiniones de las partes interesadas y de incorporar los juicios y valores de diferentes stakeholders. Pedimos a 250 expertos que completaran un cuestionario para determinar prioridades entre los diferentes criterios relacionados con las carreteras a lo largo de su ciclo de vida. La encuesta incluyó expertos de centros de investigación de transporte, sector público, especialistas de organismos internacionales (Banco Mundial, Banco Europeo de Inversiones, entre otros), así como profesores, investigadores e ingenieros de diferentes lugares del mundo. A los encuestados se les pedía indicar sus preferencias para cada criterio, presentado en comparación con otro criterio y marcar sus opciones, utilizando la escala de REMBRANDT. El cuestionario preguntaba explícitamente sobre la importancia de los criterios, con independencia del contexto del proyecto y de la magnitud de los impactos, evitando así una doble contabilización. El 
proyecto específico a ser evaluado no fue mencionado en el cuestionario para evitar el sesgo.

Sobre la base de su visión personal y de experto, los encuestados respondían acerca de la importancia relativa de criterios económicos, ambientales y sociales definidos para este caso. Se obtuvo así el peso de cada criterio mediante el uso de la técnica de REMBRANDT - ver Figura. 2. Los responsables de tomar decisiones mostraron una fuerte preferencia sobre el criterio de 'ahorro de costos de accidentes' con respecto a otros criterios. En contraste, para los aspectos que tienen que ver con el medio ambiente y con lo social, las diferencias no fueron significativas. Se encontró una preferencia débil por los criterios sociales sobre los ambientales y económicos. Esto implica que el empeoramiento de un criterio de sostenibilidad podría ser compensado por la mejora de un aspecto social. Sin embargo, la magnitud del impacto es importante en la evaluación final de la sostenibilidad.

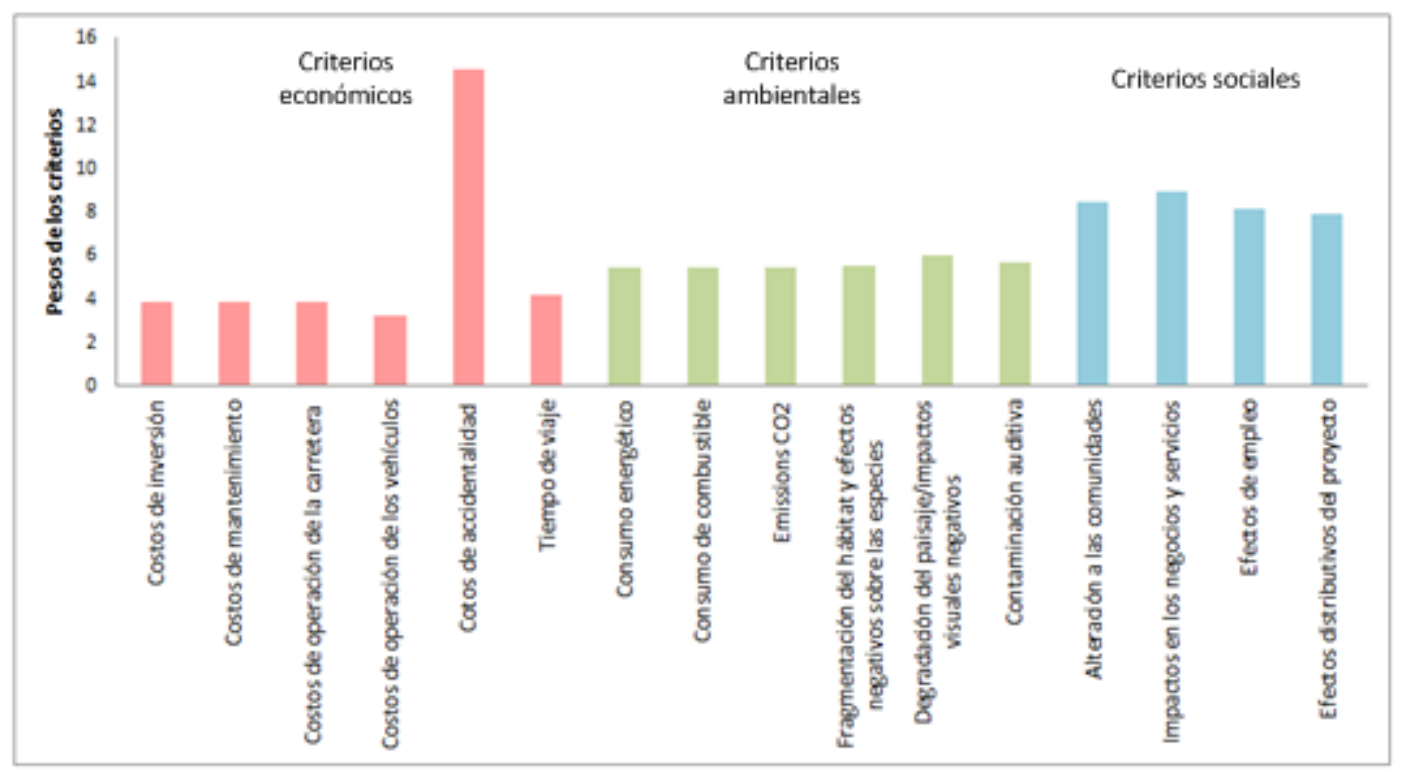

Fig. 2 - Pesos de los criterios, resultados de la encuesta de expertos

Por último, el nivel de consenso entre el panel de expertos se estimó mediante el uso de la prueba estadística basado en la técnica de validación cruzada desarrollada por nuestra metodología. Hemos dividido los datos en dos partes y hemos comparado las respuestas de los expertos encuestados de ambos grupos. Repetimos el procedimiento 1000, $10000 \mathrm{y}$ 100000 veces, y finalmente obtuvimos una distribución p-valor para cada criterio de sostenibilidad. Todos estos resultados reportaron valores superiores a 0.05 , lo que nos permite llegar a la conclusión de que el nivel de consenso es lo suficientemente bueno por lo que la aplicación de un método Delphi no es necesaria en este caso. Como lo indica el proceso estadístico, asumimos los pesos de los criterios derivados de la encuesta como los CWs finales. Estos pesos se ajustaron posteriormente con el nivel de severidad obtenido de la primera parte del caso de estudio. Finalmente, calculamos un IW para cada criterio aplicando la Ecuación 1. Los resultados se muestran en la última columna de la Tabla 2. 


\section{CONCLUSIONES}

En el presente trabajo, hemos propuesto una nueva, sencilla y transparente metodología para ayudar a los tomadores de decisiones a determinar de manera efectiva las ponderaciones de los diferentes criterios para evaluar un proyecto de transporte o para seleccionar una alternativa.

Este enfoque obtiene los pesos considerando por separado la sensibilidad de los criterios en el ámbito geográfico o contexto en el que se encuentra el proyecto, y la importancia relativa de los diferentes criterios basada en juicios o valoraciones de expertos. Para mostrar la aplicabilidad de la metodología propuesta en este trabajo, se aplicó a un proyecto de carretera real en España. Este ejercicio demostró que el método es adecuado en el propósito de la obtención de los pesos de los diferentes criterios de sostenibilidad en proyectos viales.

Este ejemplo muestra que el método de ponderación propuesto tiene una serie de ventajas incluyendo: (i) su simplicidad, lo que facilita su comprensión y el uso de este método para aplicaciones prácticas; (ii) su flexibilidad y la capacidad de replicación, lo que permite que pueda adaptarse a diferentes aplicaciones del mundo real (por ejemplo, tiene la ventaja de permitir, el uso de los resultados de los juicios comparativos y las preferencias para muchos proyectos de carreteras); (iii) su capacidad de aumentar la eficiencia, el rigor y la objetividad del proceso de obtención de pesos en un MCDA.

Las investigaciones futuras deberían seguir comprobando la aplicabilidad y la utilidad de la metodología propuesta por ejemplo aplicándolo a otros casos de estudio y comparando los resultados con otros enfoques para el establecimiento de los coeficientes de ponderación. También se sugiere aplicar el método a proyectos que ya se han sido evaluados, a fin de comparar las diferencias entre resultados. De esta manera algunas preguntas pertinentes podrían ser respondidas (como lo que habría tendría pasado si hubiéramos procedido de acuerdo a otro método de obtención de los pesos con la selección final de la mejor alternativa).

Finalmente, se espera que el nuevo método de ponderación tenga un gran potencial para ser implementado en los procesos de evaluación multicriterio destinados a evaluar la sostenibilidad. Sin embargo, múltiples investigaciones deben ser llevadas a cabo con el fin de resolver otros problemas conocidos del MCDA tales como la agregación inter-temporal de criterios medio ambientales, sociales y económicos, mejorando así la evaluación a lo largo del ciclo de vida.

\section{REFERENCIAS}

BUENO, P.; VASSALlO, J. y CHEUNG, K. (2015). Sustainability Assessment of Transport Infrastructure Projects: a Review of Existing Tools and Methods. Transport Reviews 35(5), pp. 1-28. 
BROWNE, D. y RYAN, L. (2011). Comparative analysis of evaluation techniques for transport policies. Environmental Impact Assessment Review 31(3), pp. 226-233.

CHEN, S.; LENG, Y.; MAO, B. y LIU, S. (2014). Integrated weight-based multi-criteria evaluation on transfer in large transport terminals: A case study of the Beijing South Railway Station. Transportation Research Part A: Policy and Practice 66(1), pp. 13-26.

CHEN, Y.; YU, J. y KHAN, S. (2013). The spatial framework for weight sensitivity analysis in AHP-based multi-criteria decision making. Environmental Modelling and Software 48, pp.129-140.

DASGUPTA, S. y TAM, E.K. (2005). Indicators and framework for assessing sustainable infrastructure. Canadian Journal of Civil Engineering 32(1), pp. 30-44.

GILMOUR, D.; BLACKWOOD, D.; BANKS, L. y WILSON, F. (2011). Sustainable development indicators for major infrastructure projects. Proceedings of the Institution of Civil Engineers-Municipal Engineer 164(1), pp. 15-24.

GÜHNEMANN, A.; LAIRD, J.J. y PEARMAN, A.D. (2012). Combining cost-benefit and multi-criteria analysis to prioritise a national road infrastructure programme. Transport Policy 23, pp. 15-24.

HOBBS, B.F. y HORN, G.T. (1997). Building public confidence in energy planning: a multimethod MCDM approach to demand-side planning at BC gas. Energy Policy 25(3), pp. $357-375$.

INIESTRA, J.G. y GUTIÉRREZ, J.G. (2009). Multicriteria decisions on interdependent infrastructure transportation projects using an evolutionary-based framework. Applied Soft Computing 9(2), pp. 512-526

JANIC, M. (2003).Multicriteria evaluation of high-speed Rail, transrapid maglev and air passenger transport in Europe. Transportation Planning and Technology 26(6), pp. 491512.

LINSTONE, H. y TUROFF, M. (1975). The Delphi Method: tecniques and applications. 2nd edition. Addison-Wesley. 620p.

MUNDA, G. (1995). Multicriteria evaluation in a fuzzy environment: theory and applications in ecological economics. Physica-Verlag. 255p.

OLSON, D.L.; FLIEDNER, G. y CURRIE, K. (1995). Comparison of the REMBRANDT system with analytic hierarchy process. European Journal of Operational Research 82(3): $522-539$.

REZAEI, J. (2015). Best-worst multi-criteria decision-making method. Omega 53, pp. 4957. 
SAYERS, T.M.; JESSOP, A.T. y HILLS, P.J. (2003). Multi-criteria evaluation of transport options-flexible, transparent and user-friendly?. Transport Policy 10(2), pp. 95-105.

TSAI, C. y CHANG, A.S. 2012. Framework for developing construction sustainability items: the example of highway design. Journal of Cleaner Production 20(1), pp. 127-136.

TUDELA, A.; AKIKI, N. y CISTERNAS, R. (2006). Comparing the output of cost benefit and multi-criteria analysis. Transportation Research Part A: Policy and Practice 40(5), pp. 414-423.

WANG, Y.J. (2015). A fuzzy multi-criteria decision-making model based on simple additive weighting method and relative preference relation. Applied Soft Computing 30: 412-420

WIBOWO, S. y DENG, H. (2011). Intelligent Decision Support for Criteria Weighting in Multicriteria Analysis for Evaluating and Selecting Cargo Ships under Uncertainty. International MultiConference of Engineers and Computer Scientists, 12-14 Marzo 2011, pp.1411-1416. Hong Kong, China. 